\title{
ALGUNAS PLANTAS INTERESANTES DE LA PROVINCIA DE CORDOBA
}

\author{
E. RUIZ DE CLAVIJO \& J. MUÑOZ
}

\begin{abstract}
RESUMEN: Se incluyen en esta nota 38 táxones recolectados en la provincia de Córdoba que representan una aportación al conocimiento florístico de la misma y de Andalucía Occidental.
\end{abstract}

SUMmaRY: In this paper 38 taxa collected in the province of Córdoba are included. Most of them are new records for the province.

La mayoría de las especies que se relacionan en esta nota han sido recolectadas en la comarca del Zújar, situada al NO de la provincia de Córdoba y limítrofe con las de Badajoz y Ciudad Real. De sustrato litológico esencialmente silíceo es una zona que se encuentra aún poco estudiada desde el punto de vista florístico, siendo muy escasas las referencias bibliográficas existentes (Devesa \& Cabezudo, 1978; González Soriano, 1923; Rivas Goday, 1964).

Para cada una de las localidades de los táxones recolectados, se indican las coordenadas geográficas según la proyección UTM en cuadrículas de $10 \mathrm{Km}$ de lado. Los ejemplares testigos se encuentran depositados en el Herbario del Departamento de Botánica de la Facultad de Ciencias de Córdoba.

Isoetes setacea Lam.

Entre Hinojosa del Duque y Estación del Mármol. Arroyo de la Jarilla, (UH-06), 20.IV.1979, Díaz, Fernández \& Muñoz.

Especie anfibia que vive en charcos o lagunas que se secan durante el periodo estival. Poco frecuente en la provincia. Sobre suelos ácidos en el borde occidental de la misma.

Isoetes velata A. Braun in Bory \& Durieu

Entre Belalcázar y Estación de Belalcázar, (UH-17), 31.V.1983, Prados \& Ruiz de Clavijo.

Primera cita de este taxon para la provincia de Córdoba, presentando la misma ecología y distribución que la especie precedente.

Cheilanthes hispanica Mett.

Santa Eufemia. Pico Horcón, (UH-37), 8.VI.1981, Muñoz, Ruiz de Clavijo \& al. Ibídem, 31.V.1983, Prados \& Ruiz de Clavijo. Entre 
Belalcázar y Estación de Belalcázar, (UH-17), 31.V.1983, Prados \& Ruiz de Clavijo.

Frecuente en las fisuras de los roquedos silíceos de la Sierra Norte cordobesa y de la comarca de los Pedroches.

Asplenium billotii F.W. Schultz

Santa Eufemia. Pico Horcón, (UH-37), 8.VI.1981, Muñoz, Ruiz de Clavijo \& al. Ibídem, 31.V.1983, Prados \& Ruiz de Clavijo.

Poco frecuente en la provincia. Sobre roquedos silíceos en el NO de la comarca de los Pedroches.

Paronychia cymosa (L.) DC. in Lam.

Entre Belalcázar y Estación de Belalcázar, (UH-17), 31.V.1983, Prados \& Ruiz de Clavijo.

Especie poco frecuente, recolectada en el NO de la provincia en pedregales silíceos.

Spergula morisonii Boreau in Duchartre

Santa Eufemia. Pico Horcón, (UH-37), 8.VI.1981, Muñoz, Ruiz de Clavijo \& al.

Silicícola. Poco frecuente. En pedregales nitrificados.

Dianthus scaber Chaix subsp. toletanus (Boiss. \& Reuter) Tutin

Entre Belalcázar y Estación de Belalcázar, (UH-17), 8.VI.1981, Ruiz de Clavijo \& al.

Endemismo hispano-lusitano encontrado sobre suelos esqueléticos

muy áridos en el NO de la provincia. Poco frecuente.

Nymphaea alba L.

Entre Blazquez y Peraleda del Zaucejo. Río Zújar, (TH-75), 6.VI.1979, Muñoz, Ruiz de Clavijo \& al.

Rara en la provincia, localizada tan solo en el río Zújar.

Clematis campaniflora Brot.

Entre Belalcázar y Cábeza de Buey. Río Zújar, (UH-18), 8.VI. 1981, Muñoz, Ruiz de Clavijo \& al. Entre Belalcázar y Estación de Belalcázar. Río Guadamatilla, (UH-17), 8.VI.1981, Muñoz, Ruiz de Clavijo \& al. Ibídem, 31.V.1983, Prados \& Ruiz de Clavijo.

Taxon endémico de la Península Ibérica. Localizado exclusivamente en los márgenes de los cursos de agua al NO de la provincia. Muy poco frecuente.

Sisymbrium runcinatum Lag. ex DC.

Entre Belalcázar y Estación de Belalcázar, (UH-17), 8.Vi.1981, Muñoz, Ruiz de Clavijo \& al.

Poco frecuente. En lugares nitrificados.

Malcomia lacera (L.) DC.

Entre Hinojosa del Duque y el Viso. Río Guadamatilla, (UH-26), 21.V.1979, Muñoz \& al. Entre Hinojosa del Duque y Fuente la Lancha. Río Guadamatilla, (UH-15), Diaz. siliceos.

Poco frecuente. En zonas arenosas muy lavadas, sobre sustratos

Lepidium heterophyllum Bentham

Entre Belalcázar y Cabeza de Buey. Río Zújar, (UH-18), 8.VI.

1981, Muñoz, Ruiz de Clavijo \& al.

En lugares ruderalizados. Poco frecuente. 
Lepidium latifolium L.

Aguilar de la Frontera. Laguna de Zóñar, (UG-55), 27.VII.1983, Ruiz de Clavijo.

Especie muy rara en la provincia, localizada únicamente en los márgenes permanentemente húmedos de la laguna de Zoñar.

Rhynchosinapis hispida (Cav.) Heywood

Santa Eufemia. Pico Horcón, (UH-37), 8.VI.1981, Muñoz, Ruiz de Clavijo \& al.

En pedregales silíceos. Poco frecuente.

Crambe hispanica L.

Santa Eufemia. Pico Horcón,(UH-37), 8.VI.1981, Muñoz, Ruiz de

Clavijo \& al. Ibídem, 31.VI.1983, Prados \& Ruiz de Clavijo.

Poco frecuente. En roquedos silíceos.

Sedum brevifolium DC.

Santa Eufemia. Pico Horcón, (UH-37), 31.V.1983, Prados \& Ruiz de Clavijo.

Sobre rocas graníticas, en el NO de la provincia. Poco frecuente.

Sedum hirsutum All.

Santa Eufemia. Pico Horcón, (UH-37), 8.VI.1981, Muñoz, Ruiz de

Clavijo \& al. Ibídem, 31.V.1983, Prados \& Ruiz de Clavijo.

De hábitat similar al de la especie anterior. Poco frecuente.

Anthyllis cornicina L.

Santa Eufemia. Pico Horcón, (UH-37), 8.Vi.1981, Muñoz, Ruiz de Clavijo \& al.

Poco frecuente. En pastizales desarrollados sobre suelos silíceos.

Linum tryginum L.

Entre Belalcázar y Estación de Belalcázar, (UH-17), 31.V.1983, Prados \& Ruiz de Clavijo.

En secarrales pedregosos. Poco frecuente.

Elatine macropoda Guss.

Belalcázar. Río Guadamatilla, (UH-17), 31.V.1983, Prados \& Ruiz de Clavijo.

En zonas encharcadas.

Magydaris panacifolia (Vahl) Lange in Willk. et Lange

Santa Eufemia. Pico Horcón, (UH-37), Prados \& Ruiz de Clavijo.

En lugares sombríos. Rara.

Anagallis tenella (L.) L.

Santa María de Trassiera. Río Guadiato, (UH-30), 13.VI.1978, Dominguez \& al.

En lugares permanentemente húmedos. Poco frecuente.

Limonium echioides (L.) Miller Lucena. Laguna Amarga, (UG-53), 27.VII.1983, Ruiz de Clavijo. En suelos salinos. Poco frecuente.

Cressa cretica L.

Puente Genil. Laguna de Tíscar, (UH-34), 27.VII.1983, Ruiz de Clavijo.

Sobre suelos salinos. Poco frecuente. 
Callitriche brutia Petagna

Santa Eufemia. Pico Horcón, (UH-37), 31.V.1983, Prados \& Ruiz de Clavijo.

En lugares encharcados.

Teucrium scordium L. subsp. scordioides (Schreber) Maire \& Petitmengin

Belalcázar. Río Guadamatilla, (UH-17), 30.VI.1983, Galán \& Ruiz de Clavijo. cuente.

En suelos encharcados de los bordes de cursos de agua. Poco fre-

Hyoscyamus niger L.

Belalcázar. Estación de Zújar, (TH-97), 31.V.1983, Prados \& Ruiz de Clavijo.

Ruderal y nitrófila. Poco frecuente.

Linaria spartea (L.) Willd.

Belalcázar. Río Guadamatilla, (UH-17), 8.VI.1981, Muñoz, Ruiz de

Clavijo \& al. Santa Eufemia. Pico Horcón, (UH-37), 31.V.1983, Prados

\& Ruiz de Clavijo.

Poco frecuente. Sobre suelos arenosos silíceos.

Linaria saxatilis (L.) Chaz.

Santa Eufemia. Pico Horcón, (UH-37), 8.VI.1981, Muñoz, Ruiz de Clavijo \& al.

En lugares pedregosos silíceos. Poco frecuente.

Digitalis thapsi L.

Santa Eufemia. Pico Horcón, (UH-37), 8.VI.1981, Muñoz, Ruiz de Clavijo \& al. Ibídem, 31.V.1983, Prados \& Ruiz de Clavijo.

Especie subnitrófila poco frecuente en la provincia, encontrándose exclusivamente en el NO de la misma sobre suelos silíceos.

Jasione crispa (Pourret) Samp. subsp. mariana (Willk.) Riv.-Mart.

Santa Eufemia. Pico Horcón, (UH-37), 8.VI.1981, Muñoz, Ruiz de Clavijo \& al. Ibidem, 31.V.1983, Prados \& Ruiz de Clavijo.

Especie rupícola muy rara en la provincia, localizada únicamente en el NO de la misma. Sobre rocas silíceas.

\section{Eupatorium cannabinum L.}

Santa María de Trassierra. Alto de las Cabreras, (UH-30), 15.VIII.1977, Tormo. Santa María de Trassierra, Arroyo de. Don Lucas, (UH-30), 30.IX.1980, Ruiz de Clavijo \& Varela.

Localizada en zonas sombrías y permanentemente húmedas. Especie muy rara en la provincia.

Alisma lanceolatum With.

Belalcázar. Río Guadamatilla, (UH-17), 8.VI.1981, Muñoz, Ruiz de Clavijo \& al. Ibídem, 31.V.1983, Prados \& Ruiz de Clavijo. Entre Belalcázar y Cabeza de Buey. Río Zújar, (UH-18), 30.VI.1983, Galán \& Ruiz de Clavijo. te.

En márgenes de charcos y de cursos de agua lenta. Poco frecuen-

Baldellia ranunculoides (L.) Parl.

Entre Belalcázar y Cabeza de Buey. Río Zújar, (UH-18), 8.VI. 1981, Muñoz, Ruiz de Clavijo \& al. Belalcázar. Río Guadamatilla, (UH-17), 8.VI.1981, Muñoz, Ruiz de Clavijo \& al. Ibídem, 31.V.1983, 
Prados \& Ruiz de Clavijo.

En lugares encharcados, poco frecuente.

Potamogeton crispus L.

Belalcázar. Río Guadamatilla, (UH-17), 8.VI.1981, Muñoz, Ruiz de Clavijo \& al. Ibidem, 30.VI.1983, Galán \& Ruiz de Clavijo. Entre Belalcázar y Cabeza de Buey. Río Zújar, (UH-18), 8.VI.1981, Muñoz, Ruiz de Clavijo \& al.

Especie acuática que vive en cursos de aguas lentas, ácidas o neutras. Poco frecuente.

Potamogeton pectinatus L.

Belalcázar. Río Guadamatilla, (UH-17), 30.VI.1983, Galán \& Ruiz de Clavijo. Entre Belalcázar y Cabeza de Buey. Río Zújar, (UH-18), 30.VI.1983, Galán \& Ruiz de Clavijo.

Hábitat similar al de la especie anterior. Poco frecuente.

Najas minor All.

Entre Belalcázar y Cabeza de Buey. Río Zújar, (UH-18), 30.VI. 1983, Galán \& Ruiz de Clavijo. Belalcázar. Río Guadamatilla,(UH-17), 30.VI.1983, Galán \& Ruiz de Clavijo.

Poco frecuente. En charcas y cursos de aguas lentas.

\section{BI BL IOGRAF IA}

DEVESA, J.A. \& CABEZUDO, B. -1978- Contribución al estudio florístico del Batolito de los Pedroches (Córdoba). Lagascalia, 8:53-103.

GONZALEZ SORIANO, A. -1923- Flora de Córdoba. Bol. Real Acad. de Córdoba, 3: 93-95; 4: 93-99; 5: 49-59; 6: 77-83.

RIVAS GODAY, S. -1964- Vegetación y Flórula de la Cuenca Extremeña del Guadiana. Publicaciones Excma. Dip. Prov. de Badajoz Madrid. 